On lit bien dans les flores que les feuilles du B. pubescens portent des poils principalement sur les rejets et les jeunes plants, et que les poils sont plus rares sur les individus âgés ; mais la coïncidence entre la grandeur des feuilles et le développement des poils semble avoir passé inaperçue, de même que la réunion sur un même rameau de feuilles velues et d'autres presque glabres.

Les feuilles velues du B. pubescens sont envahies de préférence par le Melampsora betulina. On peut attribuer ce fait, soit à ce que, se développant à une époque tardive, elles offrent un tissu plus jeune, plus facilement pénétrable aux sporidies à l'époque de la dessiccation de ces dernières, soit à ce qu'elles le retiennent mieux grâce à leurs poils, soit à ce qu'étant mieux nourries, puisqu'elles ont de plus grandes dimensions, elles constituent un terrain plus favorable au développement du parasite. La seconde hypothèse me semble préférable. En effet, les feuilles velues qui naissent les premières sur les rejets ou les jeunes plants sont envahies par le Melampsora aussi bien que les suivantes, quoiqu'elles soient plus petites que ces dernières et qu'elles naissent à la même époque que les premières feuilles glabres des autres rameaux.

\title{
QUELQUES PLANTES NOUVELLES POUR LA FLORE DE FRANCE,
} par MU. J. FREYN et G. GAUTHER.

( Je commence par dire que le botaniste est essentiellement mouton de "Panurge: il suit les routes connues qui doivent lui faire cueillir "quelques plantes rares, mais classiques, qui manquent à ses collections. " Il lui arrive trop rarement de s'égarer au hasard dans les cantons nou» veaux, dans les vallées inexplorées, dont le nombre est encore grand 》 dans la Cerdagne et dans le Conflans. ”

Il ne nous est jamais arrivé de mettre le pied dans les vallées « inex- plorées $»$ des Pyrénées-0rientales, sans que les faits soient venus confirmer l'importance du conseil implicitement contenu dans ces quelques lignes que notre collègue M. Reboud adressait à M. Cosson, lors de la session extraordinaire de Prades-Mont-Louis. Si, grâce aux recherches des Gouan, des Lapeyrouse, Pourret, Bentham, aidés quelques-uns par les botanistes indigènes, Coder, Barrera et Xatard, et celles de quelques savants de notre époque, parmi lesquels nous nous plaisons plus particulièrement à citer le nom de notre ami Timbal-Lagrave; si quelques points du massif du Canigou et quelques vallées des environs immédiats de Mont-Louis ont été assez bien étudiés, que d'autres, plus nombreux encore, sont à cette heure vierges de toute empreinte de pas d'un botaniste! Que de surprises ces gorges et ces rochers inconnus ne réservent-ils pas 
à ceux qui, méprisant les sentiers battus, se décideront enfin à les visiter ! Nommer les immenses vallées de Py, de Mantet, de Carança, de Llo, d'Err, c'est indiquer suffisamment l'importance des surfaces inexplorées sur le versant français de la grande chaîne des Pyrénées-0rientales.

La vallée de Carença avait eu le don d'exercer plus spécialement sur nous une attraction puissante, tant par le pittoresque de son grandiose paysage, que par les difficultés de tous genres dont elle semblait se hérisser pour se défendre contre toute tentative. Déjà, à plusieurs reprises, nous avions essayé de l'aborder par Thues, c'est-à-dire par son débouché dans la vallée de la Tet; mais nous avions dû reculer devant les difficultés que nous avaient opposées l'escalade de rochers presque inaccessibles, l'absence de sentiers tracés et celle de ponts sur le torrent, dont il eût fallu traverser à six ou sept reprises les eaux glacées.

Nous résolûmes cependant de faire une dernière tentative, cette fois par le haut de la vallée, malgré les longueurs et les fatigues qui devaient en être la conséquence.

Nous partions dans ce but de Mont-Louis, le 9 août 1879, après avoir eu l'heureuse chance, la veille, de ramasser sur la route, presque sous les remparts de Villefranche, et aussi à la Trancade d'Ambouilla, le Sonchus aquatilis Pourret, signalé jusqu'ici seulement, pour la France, dans la localité classique du Pont de la Fous, près de Saint-Paul de Fenouillhède. Notre plan était, remontant le val d'Eynes jusqu'au col de Nourri, de prendre par la gauche, et franchissant la crête du pic d'Eynes (2786 mètres) et celle de la Fosse du Géant (2809 mètres), de descendre au-dessous du premier pic de la Vache (2812 mètres) dans une cabane de bergers que nous savions exister en aval des lacs de Carença (2266 mètres). Là nous aurions passé la nuit, pour nous trouver le lendemain, frais et dispos, au centre même des lieux que nous ambitionnions de visiter.

Mais si, dans ces montagnes sourcilleuses, le botaniste propose, le temps dispose. A peine atteignions-nous le col de Nourri, qu'un orage comme on n'en voit qu'à ces altitudes, orage oủ la pluie et la grêle nous fouettaient également, nous assaillait avec une violence inouïe. Au milieu du brouillard, nous apercevions à peine devant nous des crêtes fort mouvementées et mal connues, à tout instant frappées par la foudre. Glacés par le vent, trempés jusqu'aux os, à trois ou quatre heures de marche de notre coucher, n'était-il pas bien imprudent d'aller passer une nuit sans feu à plus de 2000 mètres d'altitude? C'était vraiment trop tenter le sort! Aussi hésitâmes-nous à peine, et pour la forme, à suivre le conseil de notre excellent guide, Michel Nou, du Vernet, qui nous engageait à descendre à l'ermitage de N.-D. de Nuria, situé sur le versant espagnol, à 1000 mètres environ, en hauteur verticale, au-dessous du 
point oủ nous nous trouvions. Jae conseil était bon : lá, pour la somme vraiment trop modique de vingt-cinq sous, nous trouvions bon gîte, bon feu, nombreuse compagnie et un souper suffisant pour nos estomacs de botanistes.

L'aube était encore loin que nous nous remettions en route; le détour de la veille allait nous valoir une rude journée de marche. Il s'agissait de franchir, entre N.-D. de Nuria et le Vernet, une distance de plus de 30 kilomètres à vol d'oiseau, coupés par quatre ascensions de 800 à 1000 mètres.

$\mathrm{Au}$ soleil levant, nous gravissions déjả les derniers lacets tracés dans les éboulis schisteux du sommet de la vallée de Nourri, qui de N.-D. (2000 mètres) conduit au pic de la Fosse du Géant (2809 mètres) par le col des Sept-Croix. Nous y recueillons une première récompense de nos fatigues: au milieu de la végétation en tout semblable à celle du sommet de la vallée d'Eynes, Iberis spathulata Berg., Alyssum cuneifolium Ten., Draba cuspidata Bieb., Galium pyrenaicum Gouan, nous pouvions récolter en abondance une plante non encore signalée, à notre connaissance, dans eette partie de la chaîne : le Veronica Nummularia Gouan; mieux encore, le Xatardia scabra Meissn., indiqué jusqu'ici exclusivement au col de Nourri, nous montrait ses rosettes radicales, que l'on ne saurait confondre avec celles de toute autre plante, quand on a récolté cette espèce dans sa localité classique. Nous ne pouvons conserver de doutes: le Xatardia scabra Meissn. croît sur les versants espagnols et français de la chaîne, depuis le col de Llo (2558 mètres) jusqu'au premier pic de la Vache.

Il nous est malheureusement impossible de nous attarder à rechercher des pieds en bel état de cette rare Ombellifère; il faut marcher. Du col que nous avions atteint, un sentier tracé sur la droite du versant français nous conduisait en une demi-heure au premier pic de la Vache. Au-dessous de nous s'étagent à diverses hauteurs les nombreux lacs de Carença et la sombre vallée qu'ils couronnent.

Que n'avons-nous les moyens et le temps d'analyser et de décrire ici l'imposant panorama de montagnes qui se déroule autour de nous, et dont les lignes principales, disposées sur divers plans, en allant de l'ouest à l'est, sont le Puigmal (2909 mètres) à la vaste croupe gazonnée, le noir pic de Sègre (2795 mètres), le Carlitte (2902 mètres) et puig Prigue (2819 mètres), avec leurs vastes champs de neige; les Cambredasses (2758 mètres), dont les rocs déchiquetés tranchent nettement sur le bleu du ciel ; le large pic de Galinas; enfin le Canigou (2783 mètres) et Costabona (2464 mètres), qui se perdent dans les vapeurs lointaines! Il faudrait ici une autre plume que la nôtre, et nous avons hâte du reste de réaliser au plus vite les promesses de notre titre. 
Des heures s'écoulent rapidement à parcourir tous les recoins des environs de Carença et de la coume de l'Enfer. Dieu sait si le temps passait vite à remplir boîtes et cartons des Senecio leucophyllus DC., Papaver suaveolens Lapeyr., Ranunculus angustifolius DC., Geum pyrenaicum Willd., Gagea fistulosa Dub., auxquels venaient plus loin s'ajouter : Ranunculus parnassifolius L., Biscutella pyrenaica Huet, Ranunculus plantagincus All., ainsi que quelques rares Saxifrages, S. pubescens Pourret, etc., et tant d'autres délicates espèces. Nous récoltions enfin quelques belles touffes d'un Erigeron confondu d'abord par nous avec l'E. uniflorus L., mais qu'une étude ultérieure plus attentive nous fait rapporter sans hésitation à l'Erigeron frigidus Boiss. Nous avons comparé avec soin notre plante à un grand nombre de pieds provenant de la sierra Nevada. Nos échantillons n'offrent aucune différence que des tiges un peu plus élevées; tous les autres caractères sont exactement ceux de l'Erigeron frigidus, notament la structure, la grandeur et la couleur vive des capitules, l'indumentum si remarquable, les feuilles et le port.

Nous pouvons donc affirmer que le rare Erigeron de la sierra Nevada appartient à la flore de France, et lui assigner pour localité : les éboulis alpins du sommet de la vallée de Carença (Pyrénées-Orientales). La plante se retrouve aussi sur les revers espagnols, oủ nous l'avons récoltée le même jour.

Parmi d'assez nombreuses Graminées formant les gazons alpins qui nous entourent, un Festuca altirait aussi vivement notre attention. Il nous paraissait, mais avec doute, se rapporter au Festuca hirsuta Host; l'un de nous ayant communiqué la plante à $\mathrm{M}$. le professeur Hackel, le savant monographe du genre Festuca, celui-ci y a reconnu le vrai Festuca Halleri All. (non Koch et mult. auct.). Cette espèce avait été entièrement méconnue par les auteurs jusqu’à Hackel, qui en a publié des exemplaires authentiques.

Là ne devait pas se borner l'intérêt de notre journée. La veille déjả, dans les éboulis du col de Nourri, nous avions pris, au milieu de l'orage, c'est-ä-dire à un moment oú nos récoltes absorbaient moins exclusivement notre attention, un petit Aronicum qui vint se représenter à nous dans les pentes raides entourant les lacs de Carença. Il existait aussi, mais plus rare, sur les rochers du sommet du pic de la Fosse du Géant, où nous l'avions constaté le matin. A première vue, on aurait pu assimiler nos échantillons à des formes réduites de l'Aronicum scorpioides DC.; mais les poils fortement glanduleux, visqueux, qui couvrent toute la plante (excepté les fleurs), les feuilles inégales dentées, entières au sommet, devaient vite nous désabuser et nous indiquer que nous possédions toute autre espèce que l'Aronicum scorpioides DC., ou même sa variélé 3. pyrenaicum Gay. Une étude plus sérieuse de notre plante nous autorise 
à la considérer comme nouvelle. La planche (1) et la description que nous joignons ici amèneront sans doute à notre opinion l'esprit de nos lecteurs.

Aronicum viscosum J. Freyn et G. Gautier.

Humile, monocephalum vel (rarius) subbrachiatum dicephalum, undique (floribus exceptis) glanduloso-viscosum. Rhizoma præmorsum, obliquum, crassum, ad collum vaginis foliorum emarcidorum confertum. Caudiculorum hornotinorum folia et caulina inferiora ovato-elliptica vel elliptica, in petiolum longum basi vaginantem contracta, obtusissima, integerrima repanda subdentata; folium subsequens oblongo-ovatum, vel oblongo-ellipticum acutiusculum breve petiolatum, summa (dua) diminuta sessilia subcordata. Capitula magna. Foliola involucri e basi ovata longe acuminata acutissima. Ligulæ oblongo-ellipticæ truncatæ, apice vel bifidæ lacinulis bidentatis, vel tridentatæ. Achenia... - $\not$. Flor. æslate.

Gallia australis in Pyreneis orientalibus : le col de Nourri, au sommet de la vallée d'Eynes, dans les éboulis schisteux; rochers du pic de la Fosse du Géant; vallée de Carença au-dessus des lacs. Altitude moyenne, 2600 mètres.

Icon. J. Freyn.

Caulis $10-20$ centimetralis. Folia basilaria usque ad $3^{\mathrm{cm}}, 5$ longa, $2^{\mathrm{cm}}, 2$ lata, caulina subminora. Petioli foliorum basilorum 5-7 ${ }^{\mathrm{cm}}$ longi. Capitulum $4-4^{\mathrm{cm}}, 5$ diametro. Phylla involucralia $15^{\mathrm{mm}}$ longa, basi usque ad $2^{\mathrm{mm}}, 5$ lata. Ligulæ $20^{\mathrm{mm}}$ longæ, supra medium $4^{\mathrm{mm}}$ latæ.

Aronicum scorpioides Koch differt indumento, habitu multo robustiore; caule elatiore magis foliato ; foliis multo majoribus grosse dentatis, caulinis nonnullis subpanduræformibus, basi cordata subamplexicaulibus; capitulis speciosis 7-8 centimetralibus, ligulis apice brevissime tridentatis subintegerrimis.

Aronicum scorpioides $\beta$ pyrenaicum G. et G., nobis ignotum, indumento plantæ nostræ simile.

Ce n'est pas, on le pense, sans regrets que nous dûmes renoncer à prolonger notre séjour dans des lieux qui, parcourus cependant sommairement, nous avaient donné des résultats si engageants. Mais les nécessités de la route nous dominaient : le soleil était déjà haut sur nos têtes, et il aurait disparu depuis longtemps quand nous pourrions atteindre le lieu de notre halte du soir. A partir du deuxième pic de la Vache, nous sortions des roches calcaires pour entrer dans le granit, c'est-à-dire dans des lieux probablement plus pauvres en bonnes espèces. Signalons toutefois une localité nouvelle de deux plantes rares dans la région qui nous occupe, les

(1) Voyez planche I de ce volume. 
Viola cenisia L. et Astragalus aristatus l'Hérit. : la première dans la vallée de Mourrens, la seconde sur le revers espagnol du col de Porteille ou de Camp-Magré. Nous pressions donc le pas sans trop craindre de laisser inaperçues des plantes bien intéressantes, et par le col de Mourrens et celui de Porteille (2478 mètres) au sommet de la vallée de Mantet, le col de Mantet (1760 mètres), les villages de Py (1000 mètres) et de Sahorre (667 mètres), nous étions enfin au Vernet, à neuf heures et demie du soir, épuisés de fatigue, mais plus satisfaits de notre journée que nous ne l'avions été de longtemps.

La liste complète des espèces récoltées par nous dans la vallée de Carença ou les environs présentera, croyons-nous, quelque intérêt ; nous la donnons done intégralement :

Campanula rotundifolia L.

Adonis pyrenaica DG.

Ammi daucifolium Scop.

Saxifraga palmata Lapeyr.

Lychnis alpina $\mathrm{L}$.

Galium cometerrhizon Lapeyr.

Veratrum album $\mathbf{L}$.

Gentiana lutea L.

Scutellaria alpina L.

Senecio Tournefortii Lap.

Cherleria sedoides $\mathbf{l}$.

Poa alpina $\mathbf{L}$.

Aquilegia cyclophylla Timb.-Lagr.

Potentilla frigida Vill.

Seseli nanum Léon Duf.

Polygala pyrenaica?

Allium schœnoprasum L.

Ranunculus montanus Will.

Meum athamanticum Jacq.

Potentilla Halleri Ser.

Saxifraga bryoides L.

- muscoides Wulf.

Cerastium lanatum Lamk.

Crepis pygmæa $\mathbf{L}$.

Cardamine amara L.

Saxifraga ascendens L.

- ajugæfolia L.

Pinus uncinata Ram.

Astragalus aristatus l'Hérit.

Ranunculus bupleurifolius Lapeyr.

Myosotis alpestris L.

- pyrenaica Pourret.

Viola valderia DC.

Potentilla nivalis Lapeyr.
Saxifraga stellaris L.

- muscoides var. compacta G. et G.

- oppositifolia L.

- pubescens Pourret.

Festuca glauca Schrad.

- Halleri All.

- longifolia Schrad.

Dianthus ferrugineus Pourret.

Muscari comosum Mill.

Ervum tetraspermum L.

Athamanta crithmoides Lapeyr.

Saxifraga mixta Lapeyr.

- Clusii Gouan.

Alchemilla pyrenaica Desf.

Stachys recta $\mathbf{L}$.

Iberis Garrexiana All.

- spathulata Berg.

Thymus angustifolius Pers.

Scleranthus perennis $\mathbf{L}$.

Saxifraga capitata Lapeyr.

Jasione humilis Pers.

Saxifraga geranioides L.

Epilobium alsinæfolium Vill.

Pedicularis pyrenaica Gay.

Alyssum cuneifolium Ten.

Draba cuspidata Bieb.

Phyteuma pauciflorum I.

Primula latifolia Lapeyr.

Arenaria striata L.

Trifolium alpinum $\mathrm{L}$.

Alsine Bauhinorum Gay.

Galium pyrenaicum Gouan.

Veronica Nummularia Gouan.

Gentiana Burseri Lapeyr. 
Gentiana nivalis $\mathbf{L}$.

Achillea pyrenaica L.

Pirola uniflora L.

Xatardia scabra Meissn.

Senecio leucophyllus DC.

Ranunculus angustifolius DC.

Silene acaulis $\mathbf{L}$.

Viola alpestris Jord.

Poa brevifolia DC.

Dianthus deltoides L.

- attenuatus Lm.

Oxyria digyna Campd.

Geum pyrenaicum Willd.

Gagea fistulosa Dub.

Ranunculus parnassifolius L.

Eryngium Bourgati Gouan.

Potentilla minima Hall.

Carduus carlinoides Gouan.

Saxifraga aizoides L.

Sideritis alpina Pourret.

Aira flexuosa L.

Calamagrostis montana Host.

$\Lambda$ rabis alpina $\mathrm{L}$.

Gentiana alpina $\mathbf{L}$.

Acinos alpina Jord.
Biscutella pyrenaica Huet.
Ranunculus plantagineus All.
Erigeron uniflorus L.
- frigidus Boiss. et R.
Aronicum viscosum J. Fr. et Gaut.
Senecio Tournefortii Lapeyr.
Peucedanum Ostruthium Koch.
Helianthemum obscurum Pers.
Veronica fruticulosa L.
- bellidioides Gouan.
Armeria alpina L.
Oxytropis campestris DC.
Phaca astragalina L.
Papaver suaveolens Lapeyr.
Astragalus aristatus L'Hérit.
Linaria alpina L.
Draba aizoides L.
Rhododendron ferrugineum L.
Hutchinsia alpina R. B.
Luzula Desvauxii Kunth.
Dryas octopetala L.
Cerastium pyrenaicum Gay.
Galeopsis pyrenaica Bartl.

Tels ont été les principaux résultats d'une course faile trop rapidement et dans des conditions atmosphériques si défavorables. Nous nous estimerions heureux si cette note, en altirant de nouveau l'attention des botanistes explorateurs, contribuait un jour à élargir le cercle de nos connaissances sur l'extrémité orientale de la chaîne pyrénéenne.

\section{NOTES SUR QUELQUES PLANTES CRITIQUES OU PEU COMMUNES, par M. Antoine LE GRAND.}

En opérant l'intercalation de nombreux paquets accumulés depuis longtemps, quelques espèces ou formes dignes d'être remarquées, comme inédites ou intéressantes pour les localités nouvelles où elles ont été récoltées, se sont présentées. J'ai pensé que des indications semblables ne seraient pas tout à fait dénuées d'intérêt et pourraient trouver place dans notre Bulletin. Le botaniste qui, surchargé de travaux spéciaux, se trouve à son grand regret détourné de ses études de prédilection, empêché de se livrer même aux excursions si fécondes en observations et en trouvailles intéressantes, peut encore parfois se dédommager en glanant dans les plantes sèches, dans de vieilles récoltes d'autrefois, oủ souvent quelque distinction d'espèce ou de forme restée douteuse ne s'éclaircit que plus tard. 


\section{$2 \mathrm{BHL}$ Biodiversity Heritage Library}

Freyn, Josef Franz and Gautier, Gaston. 1881. "Quelques Plantes Nouvelles Pour La Flore De France." Bulletin de la Société botanique de France 28, 46-52. https://doi.org/10.1080/00378941.1881.10827965.

View This Item Online: https://www.biodiversitylibrary.org/item/8651

DOI: https://doi.org/10.1080/00378941.1881.10827965

Permalink: https://www.biodiversitylibrary.org/partpdf/158712

\section{Holding Institution}

Missouri Botanical Garden, Peter H. Raven Library

\section{Sponsored by}

Missouri Botanical Garden

\section{Copyright \& Reuse}

Copyright Status: Public domain. The BHL considers that this work is no longer under copyright protection.

This document was created from content at the Biodiversity Heritage Library, the world's largest open access digital library for biodiversity literature and archives. Visit BHL at https://www.biodiversitylibrary.org. 